\title{
Adaptive WENO Schemes for Singular in Space and Time Solutions of Nonlinear Degenerate Reaction-Diffusion Problems
}

\author{
Stefka Dimova ${ }^{1, a}$ and Yonita Mihaylova ${ }^{1, b}$ \\ ${ }^{1}$ Faculty of Mathematics and Informatics, Sofia University "St. Kliment Ohridski", Bulgaria
}

\begin{abstract}
The numerical solution of nonlinear degenerate reaction-diffusion problems often meets two kinds of difficulties: singularities in space - finite speed of propagation of compact supports' initial perturbations and possible sharp moving fronts, where the solution has low regularity, and singularities in time - blow-up or quenching in finite time. We propose and implement a combination of the sixth-order WENO scheme of Liu, Shu and Zhang [SIAM J.Sci.Comput. 33, 939-965 (2011)] with an adaptive procedure to deal with these singularities. Numerical results on the mathematical model of heat structures are shown.
\end{abstract}

\section{Introduction}

It is well known that the solutions of nonlinear evolution problems may develop singularities even from smooth initial data. These singularities arise from the nonlinear mechanism of the modeled process.

One of the challenges to deal with is the localization of the process in space due to the finite speed of propagation of the compact supports' initial perturbations. Another challenge is the possible singularity in time - blow-up of the solution [3]. On the model of heat structures [2] - reaction-diffusion equations with power nonlinearities, we show how to deal with these challenges. To construct a numerical scheme, which has high order accuracy in the smooth regions and nonoscillatory behavior near to the sharp fronts of the solution, the sixth-order WENO procedure of Liu, Shu and Zhang [1] is used. To deal with the three types of the blow-up behavior of the solutions, namely, regional, total and single-point blow-up, we use the fact that the self-similar blow-up solutions of this model are attractors of its solutions for large classes of initial data. An adaptive procedure, consistent with the self-similarity law, is implemented in the cases of single-point blow-up (decreasing the step-size in space) and total blow-up (increasing the step-size). The high accuracy and the nonoscillatory behavior of the implemented numerical schemes are illustrated for all three cases of blow-up.

The outline of the paper is as follows. Section 2 describes the problem on which the proposed technique is explained. The main steps of the implemented WENO algorithm are summarized in Section 3. The strategy of the mesh adaptation is exposed in Section 4. Section 5 shows the numerical results for the three typical cases of blow-up.

\footnotetext{
ae-mail: dimova@fmi.uni-sofia.bg

be-mail: yonitamihaylova@yahoo.com
} 


\section{The problem}

The proposed numerical method is presented on the simplest 1D heat model [2]

$$
\begin{gathered}
u_{t}=\left(u^{\sigma} u_{x}\right)_{x}+u^{\beta}, x \in \mathbb{R}_{+}, t>0, \\
u_{x}(t, 0)=0, \quad u(t, \infty)=0, \quad u^{\sigma} u_{x}=0 \text { if } u=0 .
\end{gathered}
$$

Here $u(t, x)$ is the temperature of the heat-conducting medium, whose heat-conductivity coefficient $u^{\sigma}, \sigma>0$, and the volume source $u^{\beta}, \beta>1$, are power functions of the temperature. A variety of real processes are described well by this model - from combustion processes in hot plasmas to that in stars. It is well known [3], that for $\beta>1$ the equation (1) admits blow-up self-similar solutions (s.-s.s.) $u_{s}(t, x)$ of the kind

$$
\begin{gathered}
u_{s}(t, x)=\varphi(t) \theta(\xi)=\left(1-t / T_{0}\right)^{-\frac{1}{\beta-1}} \theta(\xi), \\
\xi=x / \psi(t)=x\left(1-t / T_{0}\right)^{-\frac{\beta-\sigma-1}{2(\beta-1)}},
\end{gathered}
$$

where $T_{0}<\infty$ is the blow-up time and $\varlimsup_{t \rightarrow T_{0}^{-}} \sup _{x \in \mathbb{R}^{N}} u_{s}(t, x)=+\infty$. The space $x$ and the time $t$ (through the function $\psi(t)$ ) are connected in the similarity variable $\xi$. The self-similar function $\theta(\xi)$ satisfies the nonlinear degenerate boundary-value problem:

$$
\begin{gathered}
-\left(\theta^{\sigma} \theta^{\prime}\right)^{\prime}+\frac{\beta-\sigma-1}{2(\beta-1) T_{0}} \xi \theta^{\prime}+\frac{1}{(\beta-1) T_{0}} \theta-\theta^{\beta}=0, \\
\theta^{\prime}(0)=0, \quad \theta(\infty)=0, \quad \theta^{\sigma} \theta^{\prime}=0, \text { if } \theta=0 .
\end{gathered}
$$

It is seen from (3) that the s.-s.s. $u_{s}(t, x)$ corresponds to the initial data $\theta(x): u_{s}(0, x)=\theta(x)$.

Without loss of generality (because of the similarity) we can put $(\beta-1) T_{0}=1$ in (5). Then the blow-up time of the solution of the problem (1), (2) with initial data $\theta(x)$, found in the computations, must be close to $T_{0}=1 /(\beta-1)$. This will serve as an internal criteria for the accuracy of solving both the self-similar problem (5), (6) and the evolution problem (1), (2).

Because the s.-s.s. $u_{s}(t, x)$ is an attractor of the solutions of the problem (1), (2) for wide classes of initial data (see [3] and the references therein), it determines the basic regimes of combustion of the nonlinear medium. They depend on the relation between the powers $\sigma$ and $\beta$, i.e., on the competition between the heat diffusion and the source.

If $\beta=\sigma+1$, at the asymptotic stage $t \rightarrow T_{0}^{-}$( $t$ tends to $T_{0}$ from below) the solution blows up at every point of a finite region and this is called regional blow-up. This is the unique case, when the exact solution of the problem (5), (6) is known:

$$
\theta(\xi)=\left(\frac{2(\sigma+1)}{\sigma+2} \cos ^{2} \frac{\pi \xi}{L_{s}}\right)^{1 / \sigma}, L_{s}=2 \pi \sqrt{\frac{\sigma+1}{\sigma}}, \theta\left(\frac{L_{s}}{2}\right)=0 .
$$

The process is localized on the so called fundamental length $L_{s}$.

If $\beta<\sigma+1$, at the asymptotic stage $t \rightarrow T_{0}^{-}$the solution blows up at the whole space: for fixed $\xi$ and $t \rightarrow T_{0}^{-}: x=\xi\left(1-\frac{t}{T_{0}}\right)^{\frac{\beta-\sigma-1}{2(\beta-1)}} \rightarrow \infty$. The process is not localized, this is called total blow-up.

If $\beta>\sigma+1$, for fixed $\xi$ and $t \rightarrow T_{0}^{-}: x=\xi\left(1-\frac{t}{T_{0}}\right)^{\frac{\beta-\sigma-1}{2(\beta-1)}} \rightarrow 0$. The process is localized, this is called single point blow-up.

The condition $\sigma>0$ is a necessary and sufficient condition for a finite speed of the heat propagation to take place for finite support initial perturbations in an absolutely cold medium. The condition $\beta>1$ is a necessary condition for blow-up. So the two challenges - the localization of the process in space and the singularity in time, are present in the problem (1), (2). 


\section{WENO schemes for nonlinear degenerate reaction-diffusion equations}

The idea of the method and the main steps for its implementation will be given on a more common $1 \mathrm{D}$ equation

$$
u_{t}=(b(u))_{x x}+q(u), u \geq 0, b(u) \geq 0, q(u) \geq 0 .
$$

Let $\cdots<x_{i}<x_{i+1}<x_{i+2}<\cdots, x_{i+1}-x_{i}=\Delta x$ be a regular mesh in space. The aim is to construct a semidiscrete approximation of equation (8)

$$
\frac{d u_{i}(t)}{d t}=\frac{\hat{f}_{i+\frac{1}{2}}-\hat{f}_{i-\frac{1}{2}}}{\Delta x^{2}}+q\left(u_{i}(t)\right),
$$

such that

$$
\frac{\hat{f}_{i+\frac{1}{2}}-\hat{f}_{i-\frac{1}{2}}}{\Delta x^{2}}=\left.(b(u))_{x x}\right|_{x=x_{i}}+O\left(\Delta x^{k}\right)
$$

is a high order approximation of $(b(u))_{x x}$ at the points $x=x_{i}$, where the solution is smooth, and generates nonoscillating approximations, where the solution or its derivatives are discontinuous. Nonoscillating approximations means, that the oscillations are of order $O\left(\Delta x^{k}\right)$. For such approximation of the second derivative we use the technique proposed in [1]. The main steps of the implemented algorithm are as follows.

1. Use a symmetric 6-point big stencil, $S=\left\{x_{i-2}, \ldots, x_{i+3}\right\}$ to approximate the numerical flux $\hat{f}_{i+\frac{1}{2}}$ and construct linear scheme of order $k=6$, which is stable, has the desired order for smooth solutions, but should be oscillatory at the points of discontinuity.

2. Use three consecutive four-point small stencils $S_{m}=\left\{x_{i-2+m}, \ldots, x_{i+1+m}\right\}, m=0,1,2$, and construct linear schemes of fourth order of accuracy and numerical fluxes $\hat{f}_{i+\frac{1}{2}}^{(m)}$.

3. Find the linear weights, the constants $d_{m}$, such that

$$
\hat{f}_{i+\frac{1}{2}}=\sum_{0}^{2} d_{m} \hat{f}_{i+\frac{1}{2}}^{(m)}
$$

4. Because in conservative approximations of the flux negative coefficients $d_{m}$ always exist [4], [1], split them into positive and negative parts by using the technique from [5], [1].

5. Find the nonlinear weights $\omega_{m}$,

$$
\hat{f}_{i+\frac{1}{2}}=\sum_{0}^{2} \omega_{m} \hat{f}_{i+\frac{1}{2}}^{(m)} .
$$

so to keep the high order of accuracy and to achieve nonoscillatory behaviour by using smoothness indicators [6], [7], [1].

The final semidiscrete problem consists of equation (9), where the fluxes (10) are used, and the initial conditions $u_{i}(0)=u_{0}\left(x_{i}\right)$. It is an ODE system of the kind $u_{t}=L(u)$. To solve it the third order TVD method of type Runge-Kutta [8] is used:

$$
u^{(1)}=u^{n}+\Delta t L\left(u^{n}\right), \quad u^{(2)}=\frac{3}{4} u^{n}+\frac{1}{4} u^{(1)}+\frac{1}{4} \Delta t L\left(u^{(1)}\right), \quad u^{(n+1)}=\frac{1}{3} u^{n}+\frac{2}{3} u^{(2)}+\frac{2}{3} \Delta t L\left(u^{(2)}\right) .
$$

The time-step $\Delta t$ is chosen so as the CFL condition for stability to be fulfilled:

$$
\max _{u}\left|b^{\prime}(u)\right| \frac{\Delta t}{\Delta x^{2}} \leq 0.4 \text {. }
$$




\section{Mesh adaptation consistent with the self-similar law}

As said in Section 2, the s.-s.s. $u_{s}(t, x)$ is an attractor of the solutions of the problem (1), (2) for wide classes of initial data $u(0, x)=u_{0}(x) \geq 0$, supp $u_{0}<\infty, x \in R_{+}$. It is natural to incorporate the structure of the s.-s.s. when designing the numerical method. The basic idea is the following. If the problem $u_{t}=L u$ admits self-similar solution of the kind $u_{s}(t, x)=\varphi(t) \theta(\xi), \xi=x / \psi(t)$, the function $\varphi(t)$ determines the amplitude of the solution and the self-similar function $\theta(\xi)$ - the "frozen" profile, the space-time structure of the solution. By using the connection between $\varphi(t)$ and $\psi(t)$ from (3),(4) we exclude the unknown blow-up time $T_{0}$ and find

$$
\xi=x \Gamma(t)^{p}, \quad \Delta \xi=\Delta x \Gamma(t)^{p}, \quad p=(\beta-\sigma-1) / 2, \quad \Gamma(t)=\frac{\max _{x} u(t, x)}{\max _{x} u_{0}(x)} .
$$

Having in mind the relation between $\xi$ and $x$ for the s.-s.s. $u_{s}(t, x)$ :

$$
\begin{array}{llll}
\text { - } & \beta<\sigma+1: & \xi \rightarrow 0 & \text { when } t \rightarrow T_{0}^{-} ; \\
\text {- } & \beta>\sigma+1: & \xi \rightarrow \infty & \text { when } t \rightarrow T_{0}^{-},
\end{array}
$$

we apply the following strategy for adaptation.

Let $\Delta x^{(k)}$ be the step in $x$ at $t=t^{k}$ and $\Delta x^{(0)}=h_{0}$.

For $\beta<\sigma+1$ (total blow-up) we choose the step in $x$ so that the step in $\xi$ be bounded from below:

$$
h_{0} / \lambda \leq \Delta \xi^{(k)}=\Delta x^{(k)} \Gamma(t)^{p}
$$

for $\beta>\sigma+1$ (single-point blow-up) we choose the step in $x$ so that the step in $\xi$ be bounded from above:

$$
\Delta \xi^{(k)}=\Delta x^{(k)} \Gamma(t)^{p} \leq \lambda h_{0} .
$$

In the computations we have used $\lambda=0.5$.

\section{Numerical results}

The methods described in Section 2 and Section 3 are implemented in $C^{++}$. Many experiments were made for problem (1), (2) with different medium parameters $\sigma>0$ and $\beta>1$. Here we present the three typical cases of regional, total and single point blow-up.

Regional blow-up. In figure 1 , left, the evolution in time of the exact solution $\theta(x)(7)$ of the self-similar problem (5), (6) for $\beta=\sigma+1=3$ is shown. The solution grows over the fundamental length (7), the fronts become very sharp and one can see only one visible negative value. The blow-up time $\tilde{T}_{0}=0.499999990$ found in the computations is an excellent approximation of the exact one $T_{0}=1 /(\beta-1)=0.5$. The computations are performed in the interval $[0,4]>\left[0, L_{s} / 2\right]$ using a constant step $\triangle x=2^{-5}$.

The real order of convergence

$$
\alpha(x)=\ln \left|\frac{u_{\text {exact }}(x)-u_{h}(x)}{u_{\text {exact }}(x)-u_{h / 2}(x)}\right| / \ln 2
$$

is tested by using the method of Runge on two embedded meshes and shown in Table 1. In the region where the solution is smooth, the order of convergence is approximately 6 (the theoretical one), and it decreases near the front, where the derivatives of the solution are discontinuous. Let us mention, the fundamental length $L_{s} / 2 \approx 2.72$ and the last two points in Table 1 are outside the interval $\left[0, L_{s} / 2\right]$. 

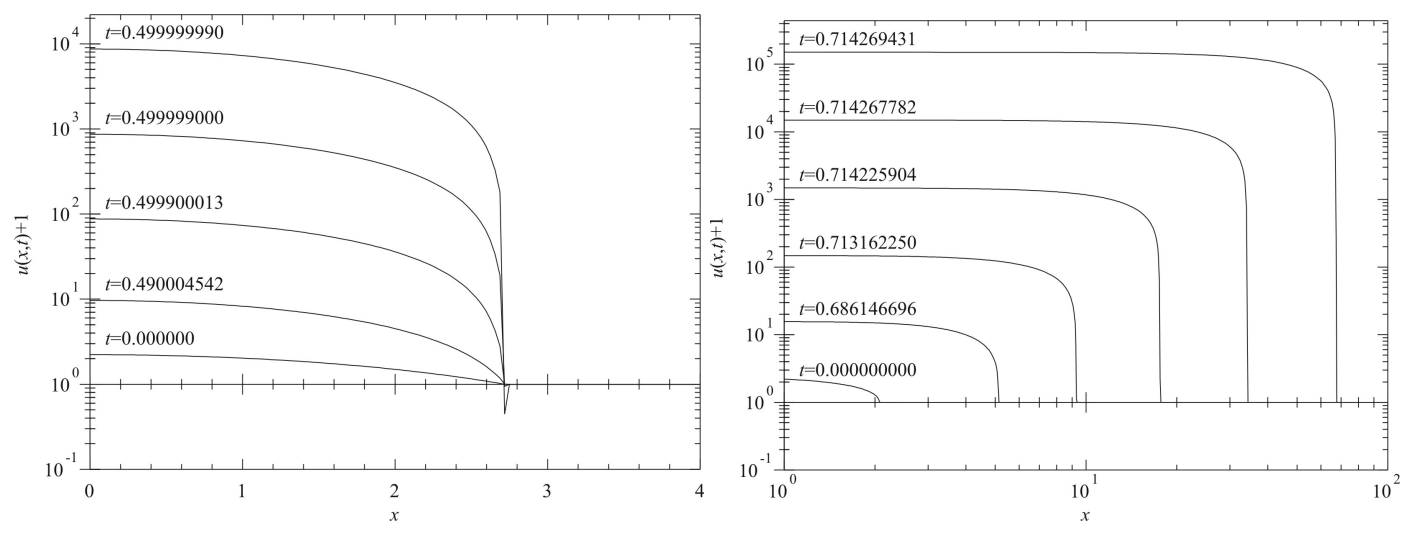

Figure 1. Evolution in time of self-similar initial data for $\beta=\sigma+1=3$, left, and for $\beta=2.4<\sigma+1=3$, right.

Table 1. $\sigma+1=\beta=3$, order of convergence, computed at time $t=0.499990, L_{s} \approx 2.72, h=1 / 32,0 \leq x \leq 4$

\begin{tabular}{lllll}
\hline$x$ & $u_{h}$ & $u_{h / 2}$ & $u_{\text {exact }}$ & $\alpha$ \\
\hline 0 & 273.8619937 & 273.8612908 & 273.8612787 & 5.88 \\
0.5 & 262.5301000 & 262.5294261 & 262.5294146 & 5.89 \\
1 & 229.4722037 & 229.4716142 & 229.4716046 & 5.95 \\
1.5 & 177.4240520 & 177.4235949 & 177.4235886 & 6.19 \\
2 & 110.6929534 & 110.6926633 & 110.6926645 & 7.99 \\
2.25 & 73.5115528 & 73.5113475 & 73.51136325 & 3.60 \\
2.5 & 34.8012804 & 34.8010643 & 34.8012322 & - \\
2.7 & 2.9217803 & 3.0779685 & 3.2727286 & - \\
2.75 & 0.059193 & -0.0019495 & 0.0 & - \\
2.95 & -0.0001131 & 0.0 & 0.0 & - \\
\hline
\end{tabular}

Total blow-up. In figure 1, right, the evolution of the self-similar function $\theta(\xi)$, found numerically as a solution of the self-similar problem (5), (6) for $\beta=2.4<\sigma+1=3$, is shown. The method for solving the $\mathrm{N}$-dimensional radially symmetric variant of the self-similar problem involving the combination of the continuous analog of the Newton method with the finite element method is described in [9]. Again the blow-up time $\tilde{T}_{0}=0.714269 \ldots$, found in the computations, is very close to the exact one, $T_{0}=1 /(\beta-1)=0 .(714285)$. The initial interval in $x$ is $\left[0 ; l_{0}=5\right]$ with $\Delta x_{0}=0.025$. When the condition (11) is violated or $l_{i}-x_{f r}(t)$ becomes less than $4 \Delta x_{i}$, we double the computational interval and the stepsize in $x: l_{i+1}=2 l_{i}, \Delta x_{i+1}=2 \Delta x_{i}$ and the intermediate points are omitted. Thus the mesh remain equidistant, which is obligatory for the conservative approximation of the flux, and the number of the computational points does not increase. In the computation shown in figure 1, right, the consecutive steps are $0.025,0.05,0.1,0.2,0.4,0.8$ and the number of mesh points is 200 . In spite of the so sharp fronts, there are no visible oscillations.

Single-point blow-up. In figure 2, left, the evolution of the cut-off self-similar function $\theta(\xi)$ found numerically as a solution of the self-similar problem (5), (6) for $\beta=3.6>\sigma+1=3$, is shown. The blow-up time $\tilde{T}_{0}=0.3846303 \ldots$, found in computations, is very close to the exact one, $T_{0}=1 /(\beta-1)=0.3846153 \ldots$ The initial interval in $x$ is $\left[0 ; l_{0}=8\right]$ with $\Delta x_{0}=0.025$. When the condition (12) is violated, we seek for an interval $\left[x_{m}, x_{l}\right]$, where the solution is already established, i.e., $\left|u\left(t_{j}, x_{i}\right)-u\left(t_{j-1}, x_{i}\right)\right|<\varepsilon$ for $x_{m} \leq x_{i} \leq x_{l}$ and some small $\varepsilon$. We throw off the mesh points 

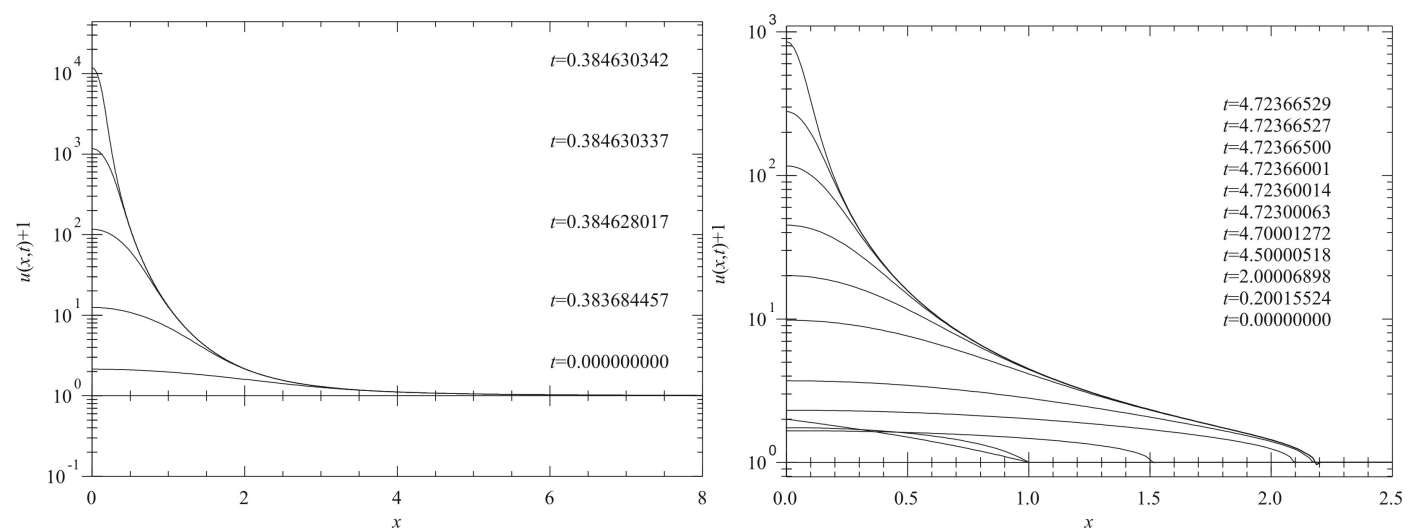

Figure 2. Evolution in time of self-similar initial data for $\beta=3.6>\sigma+1=3$ (left), and non-self-similar ones for $\beta=4>\sigma+1=3$. (right).

$x_{m+1} \leq x_{i} \leq x_{l}$ and refine the mesh in the interval $0 \leq x \leq x_{m}$, by adding midpoints between every two old mesh points. We find the values of the approximate solution in the new points by interpolation on the small stencils and continue the computations on the refined mesh in the interval $0 \leq x \leq x_{m}$. For this experiment four refinements are made up to $\Delta x_{\text {last }}=0.0015625$.

In figure 2, right, the evolution of the non-self-similar finite support initial data for parameters $\beta=4>\sigma+1=3$ is shown. There are no visible oscillations near to the finite fronts.

Conclusions. The results achieved in the 1D case promise the generalization of the technique implemented here to be successful in the radially symmetric and in the essentially $2 \mathrm{D}$ case, e.i., for the more complicated models of the heat structures [2].

\section{Acknowledgements}

The work of the first author is supported by the National Science Fund of BMSE under grant I02/9/2014 and by the Science Fund of Sofia University under grant 75/2015.

\section{References}

[1] Y. Liu, C.-W. Shu, M. Zhang, SIAM J.Sci.Comput. 33, 939-965 (2011)

[2] Stefka Dimova, Milena Dimova, Daniela Vasileva, Springer Proceedings in Mathematics \& Statistics 45, 157-184 (2013)

[3] A. A. Samarskii, V. A. Galaktionov, S. P. Kurdyumov, A. S. Mihailov, Blow-up for quasilinear parabolic equations, (De Gruyter Expositions in Mathematics 19, Valter de Gruyter, Berlin, 1995)

[4] B. Merryman, J.Sci.Comput. 19, 309-322 (2003)

[5] J. Shi, C. Hu, C.-W. Shu, J. Comput. Phys. 175, 108-127 (2002)

[6] G. Jiang, C.-W. Shu, J. Comput. Phys. 126, 202-228 (1996)

[7] C.-W. Shu, Lecture Notes in Math. 1697, 325-432 (1998)

[8] C.-W. Shu, S. Osher, J. Comput. Phys. 77, 439-471 (1988)

[9] S.N. Dimova, M.S. Kaschiev, S.P. Kurdyumov, USSR Comp. Math. Math. Phys. 29 (6), 61-73 (1989) 